\section{DETERMINING PREDICTORS FOR PERSONALIZATION OF TREATMENT OF PATIENTS WITH ENDOMETRIOID ENDOMETRIAL CANCER}

${ }^{1} \mathrm{O}$ Movchan*, ${ }^{2} \mathrm{~V}$ Svintsitskiy. ${ }^{1} \mathrm{Kyiv}$, National Cancer Institute, Kyiv, Ukraine; ${ }^{2}$ Kyiv, National cancer Institute, Kyiv, Ukraine

\subsection{6/ijgc-2021-ESG0.115}

Introduction/Background* In recent years, endometrial cancer (EC) has taken a leading position in the structure of cancer in Ukraine. The main advantages are that the disease is diagnosed in the early stages in $80 \%$ and has a good prognosis a five-year survival rate of more than 95\%. Although threequarters receive adjuvant treatment, relapses occur, on average, within the first three years. Despite the large number of studies on molecular biological markers of aggression, chemokines and their receptors, in particular CXCL12 and CXCR4, also play an important role in the biology of malignancies, the expression of which to some extent determines the progression of tumors of different genesis. High expression of CXCR4 and CXCL12 in epithelial cells is associated with a favorable course of the disease. However, high CXCR4 expression and low CXCL12 expression correlate with the aggressiveness of the tumor process. These markers will be evaluated as molecular biological predictors of endometrioid type EC aggressiveness.

Methodology To evaluate the expression level of CXCR4 and CXCL12 markers at an early stage compared to advanced forms of the disease for the personification of adjuvant treatment. We plan to include 40 patients with stage III-IV (FIGO 2009) and 40 patients with stage I-II endometrial cancer in the study.

Result(s)* Analysis with early stage and andvanced stage of EC and markers of aggressiveness CXCL12 and CXCR4 were identified. Detected negative/low expression of CXCL12 and high CXCR4 in early stages, as in advanced stages, further treatment will be recommended - radiation (remote or brachytherapy) or chemotherapy. . High CXCL12 expression and high/low CXCR4 are detected in early stage observation or appropriate protocol treatment will be recommended.

Conclusion* To analyze the level of expression of markers associated with the aggressive variant of PE in patients with the initial stage of the disease to select the type of treatment - surgery, radiation, chemotherapy or their combination to improve recurrence-free survival. The final results will be evaluated in 2023.

\section{UTERINE SEROUS CARCINOMA: ROLE OF SURGERY, RISK FACTORS AND ONCOLOGIC OUTCOMES. EXPERIENCE OF A TERTIARY CENTER}

${ }^{1} \mathrm{~A}$ Ditto*, ${ }^{1} \mathrm{~S}$ Lopez, ${ }^{1} \mathrm{~F}$ Martinelli, ${ }^{1} \mathrm{G}$ Bogani, ${ }^{2} \mathrm{~S}$ Lo Vullo, ${ }^{1} \mathrm{U}$ Leone Roberti Maggiore, ${ }^{1} \mathrm{C}$ Brusadelli, ${ }^{3} \mathrm{~B}$ Paolini, ${ }^{1} \mathrm{M}$ Ducceschi, ${ }^{1} \mathrm{M}$ Mantiero, ${ }^{1} \mathrm{~V}$ Chiappa, ${ }^{1} \mathrm{M}$ Signorelli, ${ }^{1}$ MT Evangelista, ${ }^{2} \mathrm{~L}$ Mariani, ${ }^{1} \mathrm{~F}$ Raspagliesi. ${ }^{1}$ Fondazione IRCCS Istituto Nazionale dei Tumori di Milano, Gynecologic Oncology Unit; ${ }^{2}$ Fondazione IRCCS Istituto Nazionale dei Tumori di Milano, Unit of Clinical Epidemiology and Trial Organization; ${ }^{3}$ Fondazione IRCCS Istituto Nazionale dei Tumori di Milano, Department of Pathology

\subsection{6/ijgc-2021-ESG0.116}

Introduction/Background* Uterine serous carcinoma (USC) accounts for $10 \%$ of all endometrial cancer; however, it carries the poorest prognosis, with 5 -year survival rates as low as $55 \%$. According to NCCN guidelines first-line treatment is comprehensive surgical staging by laparotomy/laparoscopy, and maximal cytoreduction to no residual disease in advanced stages. However, due to the rarity and unfavorable prognosis of the disease, available evidence is scanty and controversial on risk factors staging, adjuvant treatment, and outcome of USC.

Therefore, a retrospective study on women with USC undergoing surgery was performed to better elucidate oncologic outcomes of these patients.

Methodology This is a retrospective analysis of a prospectively collected database of consecutive patients with USC who underwent surgery between 2000-2020 at a tertiary referral center for gynecologic oncology. The primary objective of this study were progression-free (PFS) and overall survival (OS) outcomes, evaluated using Kaplan-Meier and Cox proportional hazard models.

Result(s)* 147 consecutive patients were finally included in the study. Median (IQ range) age and BMI were 66 (39-71) years and $25(39-71) \mathrm{kg} / \mathrm{m}^{2}$, respectively. Stage distribution was as follows: $67(45.6 \%)$ with early stage (stage I/II) with uterine confined disease and $80(54.4 \%)$ with advanced stages (stage III/IV) disease. The median follow-up period was 78.6 months (IQ range $=35.7-117.3$ months). The overall recurrence rate was $41 \%$ (60 patients), early-stage disease recurrence was 28.4\% (19 out of 67 ) while advanced stage disease recurrence was $51.3 \%$ (41 out of 80 ). The 5-year PFS rate was $35.0 \%$ (95\% confidence interval [CI]: 27.5-44.7\%). At multivariate analysis, age, BMI, depth of myometrial invasion, cytology, and optimal cytoreduction with postoperative residual tumor absence significantly influenced PFS. The 5-year OS rates were 46.5\% (95\% CI: 38.1-56.8]. Multivariate analysis showed that optimal cytoreduction and accuracy of retroperitoneal surgery were the only two variables influencing OS.

Conclusion* Among patients with apparent early-stage USC, peritoneal and retroperitoneal staging allows to identify those with disease harboring outside the uterus. Comprehensive surgical staging and optimal cytoreduction are the most significant prognostic factors affecting survival. Further collaborative studies are warranted in order to improve outcomes of serous endometrial cancer patients.

\section{HYSTEROSCOPIC COMPARED TO CERVICAL INJECTION FOR SENTINEL NODE DETECTION IN ENDOMETRIAL CANCER: A MULTICENTER PROSPECTIVE RANDOMIZED CONTROLLED TRIAL}

${ }^{1} \mathrm{~A}$ Ditto*, ${ }^{1} \mathrm{U}$ Leone Roberti Maggiore, ${ }^{1} \mathrm{~F}$ Martinelli, ${ }^{1} \mathrm{G}$ Bogani, ${ }^{1} \mathrm{M}$ Signorelli, ${ }^{1} \mathrm{~V}$ Chiappa ${ }^{1} \mathrm{MT}$ Evangelista, ${ }^{2} \mathrm{~J}$ Casarin, ${ }^{2} \mathrm{~F}$ Ghezzi, ${ }^{3} \mathrm{AM}$ Perrone, ${ }^{3} \mathrm{P}$ De laco, ${ }^{4} \mathrm{P}$ Scollo, ${ }^{4} \mathrm{G}$ Scibilia, ${ }^{5} \mathrm{G}$ Giorda, ${ }^{6} \mathrm{~B}$ Paolini, ${ }^{7} \mathrm{~S}$ Lo Vullo, ${ }^{7} \mathrm{~L}$ Mariani, ${ }^{1} \mathrm{~F}$ Raspagliesi. ${ }^{1}$ Fondazione IRCCS Istituto Nazionale dei Tumori di Milano, Gynecologic Oncology Unit; ${ }^{2}$ Women's and Children's Del Ponte Hospital and University of Insubria, Department of Obstetrics and Gynecology; ${ }^{3} S$. Orsola-Malpighi Polyclinic, Bologna, Italy; ${ }^{4}$ Ospedale Cannizzaro, Catania, Italy; ${ }^{5}$ C.R.O. Aviano, Italy; ${ }^{6}$ Istituto Nazionale dei Tumori | Fondazione IRCCS, Department of Pathology, Milano, Italy; 'Istituto Nazionale dei Tumori | Fondazione IRCCS, Unit of Clinical Epidemiology and Trial Organization, Milano, Italy

\subsection{6/ijgc-2021-ESG0.117}

Introduction/Background* In the last decade, sentinel lymph node mapping (SLNM) has gained a central role in endometrial cancer (EC) surgical staging. However, different technical steps of SLNM still remain object of discussione. Terofere, a randomized control trial (RCT) was conducted to compare cervical and hysteroscopic indocyanine green (ICG) 